\title{
Mechanism of Aniline Degradation by Yeast Strain Candida methanosorbosa BP-6
}

\author{
KATARZYNA MUCHA ${ }^{1}$, EWA KWAPISZ ${ }^{1}$, URSZULA KUCHARSKA ${ }^{2}$ and ANDRZEJ OKRUSZEK ${ }^{1 *}$ \\ ${ }^{1}$ Institute of Technical Biochemistry \\ ${ }^{2}$ Institute of General Food Chemistry, Technical University of Łódź, Łódź, Poland
}

Received 2 February 2010, revised 19 October 2010, accepted 29 October 2010

\begin{abstract}
The ability of some bacteria and filamentous fungi to degrade aniline and its derivatives was reported earlier in the literature. However, there was no information about the biodegradation of aniline by yeast strains. The present work is focused on yeast strain Candida methanosorbosa BP-6 which was isolated from the wastewater pool of the old dye factory "Boruta" in Zgierz by enrichment technique and identified by standard microbiological methods. We have found that strain C. methanosorbosa BP-6 readily grows in the presence of aniline and can degrade this substrate. Relatively good separation of peaks corresponding to aniline and its biodegradation intermediates allowed us their identification and quantification by HPLC methodology. We have found that major intermediates of this degradation are: catechol, cis, cis-muconic acid, muconolactone, 3-oxoadipate enol-lactone, 3-oxoadipic acid and succinic acid. Our results provide strong evidence that biodegradation of aniline by the yeast strain C. methanosorbosa BP-6 proceeds according to the intradiolic pathway.
\end{abstract}

Key words: Candida methanosorbosa yeast, aniline biodegradation, intradiolic pathway

On the long list of environmentally hazardous chemicals, prominent positions are occupied by organic nitrogen compounds and among them phenylamine, otherwise named aniline. The main source of this xenobiotic, polluting the natural environment, are sewage waters and vapors originating from chemical, tannery, cosmetic or pharmaceutical industry, utilizing aniline as one of raw materials. Due to broad range of toxicity and cancerogenicity, aniline has been subjected to stringent legislative control by US Environmental Protection Agency (1994) as well as by EC Joint Research Centre (2004). The main toxic effects of aniline start with the formation of methemoglobine in blood and are expressed by lowering of oxygen transport, anaemia, jaundice, damage of liver, spleen and lungs, and decreased blood coagulability (Agency for Toxic Substances and Disease Registry, 2009)

Aniline at very low concentrations can be readily degraded in surface waters (rivers, lakes) as discovered by Toräng et al. (2002) and Ahtiainen et al. (2003). However, the presence of the xenobiotic in wastewaters at concentration exceeding $100 \mathrm{mg} / \mathrm{ml}$ can considerably complicate its biodegradation by tradi- tional activated sludge technology (Wang et al., 2007). Because of relatively large scale of aniline release into the environment and costly procedure of its chemical remediation (Wang et al., 2007), there is an urgent need to find microorganisms able to degrade this xenobiotic. Examples of such organisms were found among various species of bacteria (Surovtseva and Volnova, 1972; Bachofer et al., 1975; Helm and Reber, 1979; Aoki et al., 1983, 1984; Lyons et al., 1984; Zeyer et al., 1985; McClure and Venables, 1986; Fujii et al., 1997; Fukumori and Saint, 1997; Zissi et al., 1997; Takeo et al., 1998; Thomas and Peretti, 1998; Kahng et al., 2000; Liu et al., 2002; Matsumura et al., 2006; Wang et al., 2007). The ability to degrade aniline and its derivatives was also found for some strains of filamentous fungi (Arjmand and Sandermann, 1985; Kremer and Sterner, 1996; Emtiazi et al., 2001). However, to the best of our knowledge there is no information in the literature about the biodegradation of aniline by yeast strains.

In our search for new microorganisms able to degrade aniline we focused our attention on the surroundings of the old (presently not operating) dye factory

\footnotetext{
* Corresponding author: A. Okruszek, Institute of Technical Biochemistry, Faculty of Biotechnology and Food Sciences, Technical University of Łódź, Stefanowskiego 4/10, 90-924 Lódź, Poland; phone: (+48) 42 6313445; fax: (+48) 42 6366618; e-mail: andrzej.okruszek@p.lodz.pl
} 


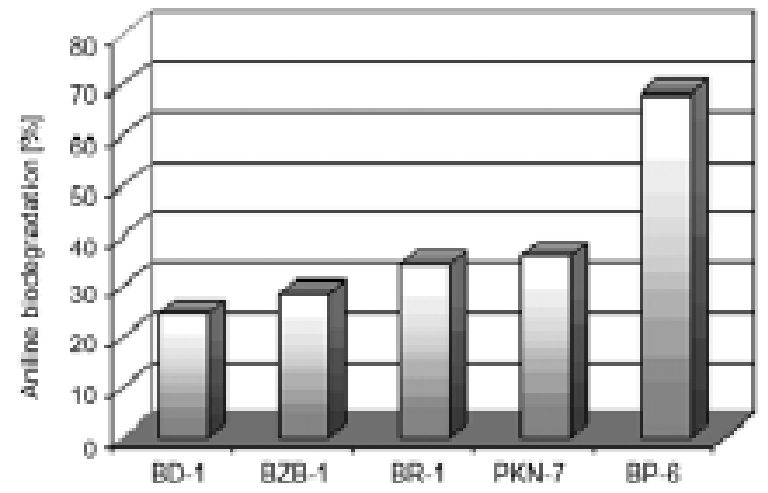

Fig. 1. Biodegradation of aniline by 5 strains of microorganisms isolated from various parts of the area surrounding old dye factory "Boruta" in Zgierz.

"Boruta" in Zgierz. Aniline was one of the basic raw materials utilized in the factory. The material for selection of microorganisms from soil and wastewater was collected in five different places of the factory area. Material from 18 samples, after appropriate dilution, was independently grown at $30^{\circ} \mathrm{C}$ on Petri dishes filled with solid King medium A (King et al., 1954) (glucose $1 \%$, bacto peptone $2 \%, \mathrm{~K}_{2} \mathrm{SO}_{4} 1 \%$, $\mathrm{MgCl}_{2} \times 6 \mathrm{H}_{2} \mathrm{O} 0.14 \%$, agar $2 \%$, pH 7.5), covered with a thin layer of aniline $(20 \mu \mathrm{l}$ per plate). The selected 18 monocultures (single colonies) were transferred into YPG slants (yeast extract $1 \%$, bacto peptone $2 \%$, glucose $2 \%$, agar $2 \%, \mathrm{pH} 7.0$ ) and maintained at $4^{\circ} \mathrm{C}$. The selected strains were tentatively identified as either bacteria or yeasts with varying abilities to degrade aniline. For further selection, each YPG slant was washed with isotonic saline to prepare inoculum for submerged shaken cultures. The cultures were run for 9 days at $30^{\circ} \mathrm{C}$ in liquid $\mathrm{AY}$ medium (yeast extract $0.2 \%$, glucose, $0.5 \%, \mathrm{NaNO}_{3} 0.05 \%$, $\mathrm{KH}_{2} \mathrm{PO}_{4} 0.075 \%, \mathrm{MgSO}_{4} \times 7 \mathrm{H}_{2} \mathrm{O} 0.022 \%, \mathrm{CaCl}_{2} \times$ $\left.6 \mathrm{H}_{2} \mathrm{O} 0.022 \%, \mathrm{pH} 5.5\right)$ in dark, in the presence of aniline $(0.5 \%)$. All strains were found to grow under these conditions with various efficiency as detected by measuring increasing cloudiness of cultures (absorption at $660 \mathrm{~nm}$, data not shown). For the best growing yeast strain isolated from sludge pool near old "Boruta" dye factory (designated by us as BP-6) and 4 best growing bacterial strains isolated from different parts of the post-industrial Zgierz area (designated as BD-1, BZB-1, BR-1 and PKN-7) the level of aniline biodegradadion was estimated by measuring of remaining aniline concentration with a Spectroquant ${ }^{\circledR}$ test at $550 \mathrm{~nm}$ (Merck) as azo-dye subsequent to azo-reaction and coupling with $\mathrm{N}-(1-$ naphtyl) ethylenediamine dihydrochloride (NEDDC) (Fig. 1). The highest level of aniline biodegradation $(68.5 \%)$ was observed for yeast strain BP-6. Therefore, this particular yeast strain was a subject of our further investigations.
Standard macro- and microscopic (500-fold magnification) observations allowed us to classify BP-6 as a yeast strain, growing in solid medium (YPG) with cream colored, smooth and glossy colonies, and in liquid medium (AY) as a suspension of oval budding cells $4-6 \mu \mathrm{m}$ in diameter. Applying diagnostic criteria described by Barnett et al., (1984) and Suye et al., (1990) led to a conclusion, that BP-6 yeast should be classified as Candida methanosorbosa. The BP-6 yeasts were found to grow readily on D-glucose, trehalose and D-galactose (slowly), whereas no fermentation of sucrose, lactose, maltose, melibiose, raffinose, inulin, melezitose or starch was observed. Also the carbon source and mineral requirements (data not shown) were in full agreement with aforementioned literature data related to $C$. methanosorbosa characterization. Ascospore formation was not observed.

From the technological point of view it was important to check the tolerance of C. methanosorbosa BP-6 towards increased concentration of aniline. Thus, several cultures of $C$. methanosorbosa BP-6 were set up in liquid medium, containing all ingredients of $\mathrm{AY}$ medium except glucose $\left(30^{\circ} \mathrm{C}, \mathrm{pH}\right.$ 5.5). In addition, the medium was supplemented with either $0.1,0.3$, $0.5,1.0,2.0,3.0$ or $4.0 \%$ of aniline as a sole carbon source. The experiments showed no decrease of biomass accumulation with increasing aniline concentration in medium within $0.1-4 \%$ range during 5 days culture. Microscopic observations, showing the morphology of cells in cultures containing increasing concentration of aniline exhibited no changes for xenobiotic concentrations up to $3 \%$.

The observed high tolerance of C. methanosorbosa BP-6 yeasts towards aniline and its efficient biodegradation prompted us to perform a study on the mechanism of this process. The available literature data show that biodegradation of aniline in various bacterial cultures may proceed either according to extradiolic or intradiolic pathway. In both cases the primary intermediate of aniline biodegradation is catechol. The extradiolic pathway, found for example for Delftia sp. (Liu et al., 2002), involves following sequence of enzymatic transformations: aniline $\rightarrow$ catechol $\rightarrow$ cis,cis-2-hydroxymuconic-6-semialdehyde $\rightarrow$ cis, cis-2-hydroxymuconic acid $\rightarrow$ 4-oxalocrotonic acid $\rightarrow$ 2-oxopent-4-enoic acid $\rightarrow$ 4-hydroxy-2-oxovaleric acid $\rightarrow$ pyruvic acid and acetaldehyde. The analogous sequence of transformations for intradiolic pathway involves: aniline $\rightarrow$ catechol $\rightarrow$ cis,cismuconic acid $\rightarrow$ muconolactone $\rightarrow$ 3-oxoadipate enol-lactone $\rightarrow 3$-oxoadipic acid $\rightarrow$ succinic acid.

For studies of the mechanism of aniline biodegradation, the submerged shaken culture of C. methanosorbosa yeasts was run in the dark at $30^{\circ} \mathrm{C}$, in liquid AY medium at $\mathrm{pH} 5.5$, in the presence of aniline $(0.5 \%)$ for 9 days. After each $24 \mathrm{~h}$ of fermentation, 
A

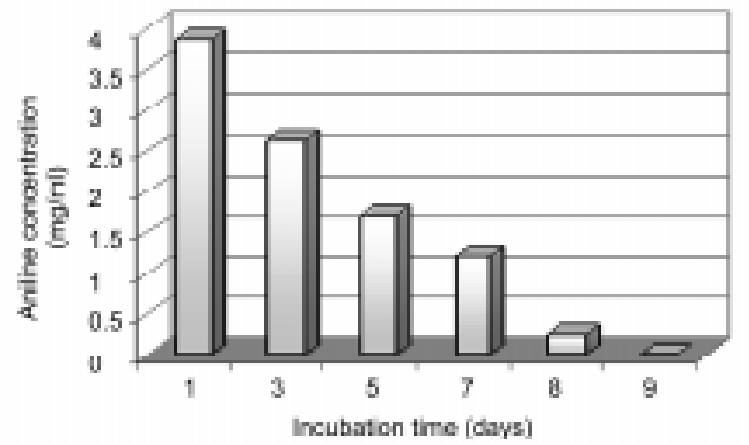

B

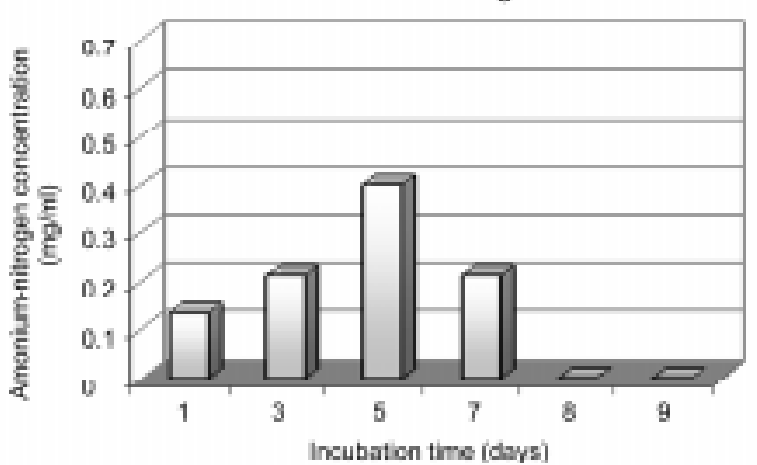

Fig. 2. Spectrophotometric determination of aniline (A) and ammonium nitrogen (B) in cell extracts obtained on consecutive days of $C$. methanosorbosa BP-6 yeast culture in AY medium in the presence of aniline $(0.5 \%)$.

a portion of the suspension was taken and yeast cells were harvested by centrifugation (30 min at $5000 \mathrm{~g}$ ). After washing with $20 \mathrm{mM}$ phosphate buffer ( $\mathrm{pH} 7.0)$, the yeast cells were sonicated $\left(5 \mathrm{~min}\right.$ at $\left.0^{\circ} \mathrm{C}\right)$ and

$$
\text { A }
$$
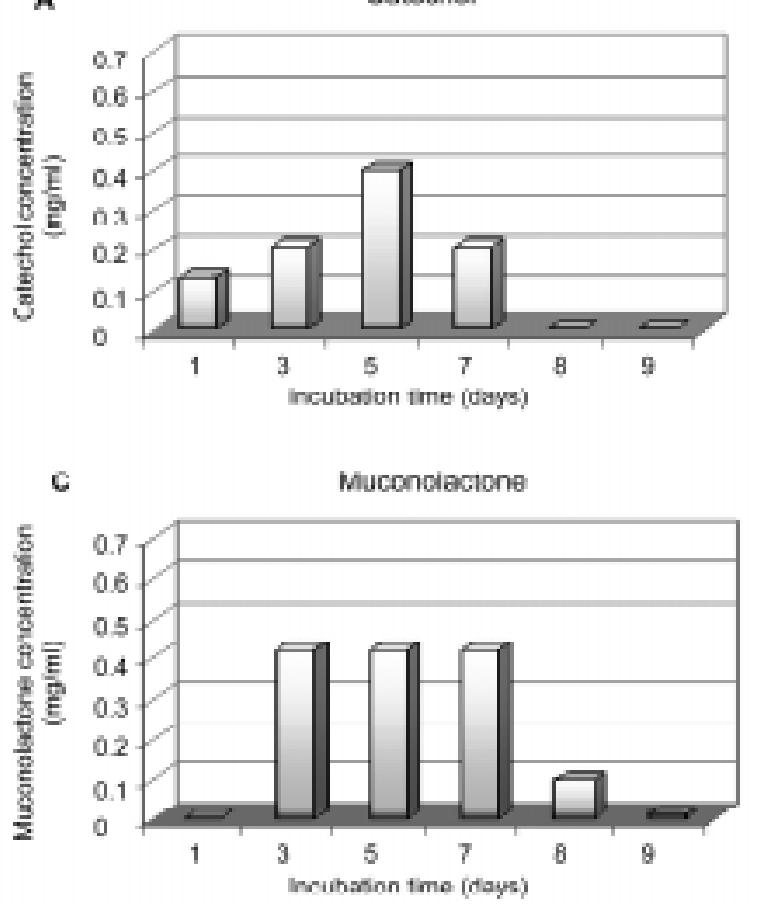

E

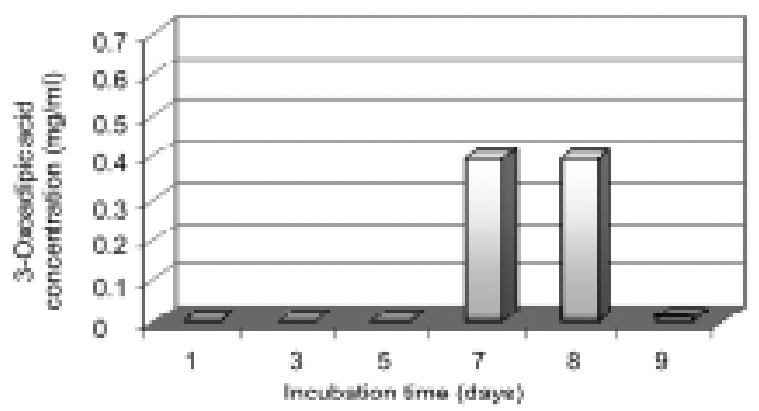

the solid parts were removed by ultracentrifugation (20 min at $19000 \mathrm{~g}$ ). The supernatants (cell extracts) were then analyzed spectrophotometrically to monitor the concentration of aniline, ammonium-nitrogen,
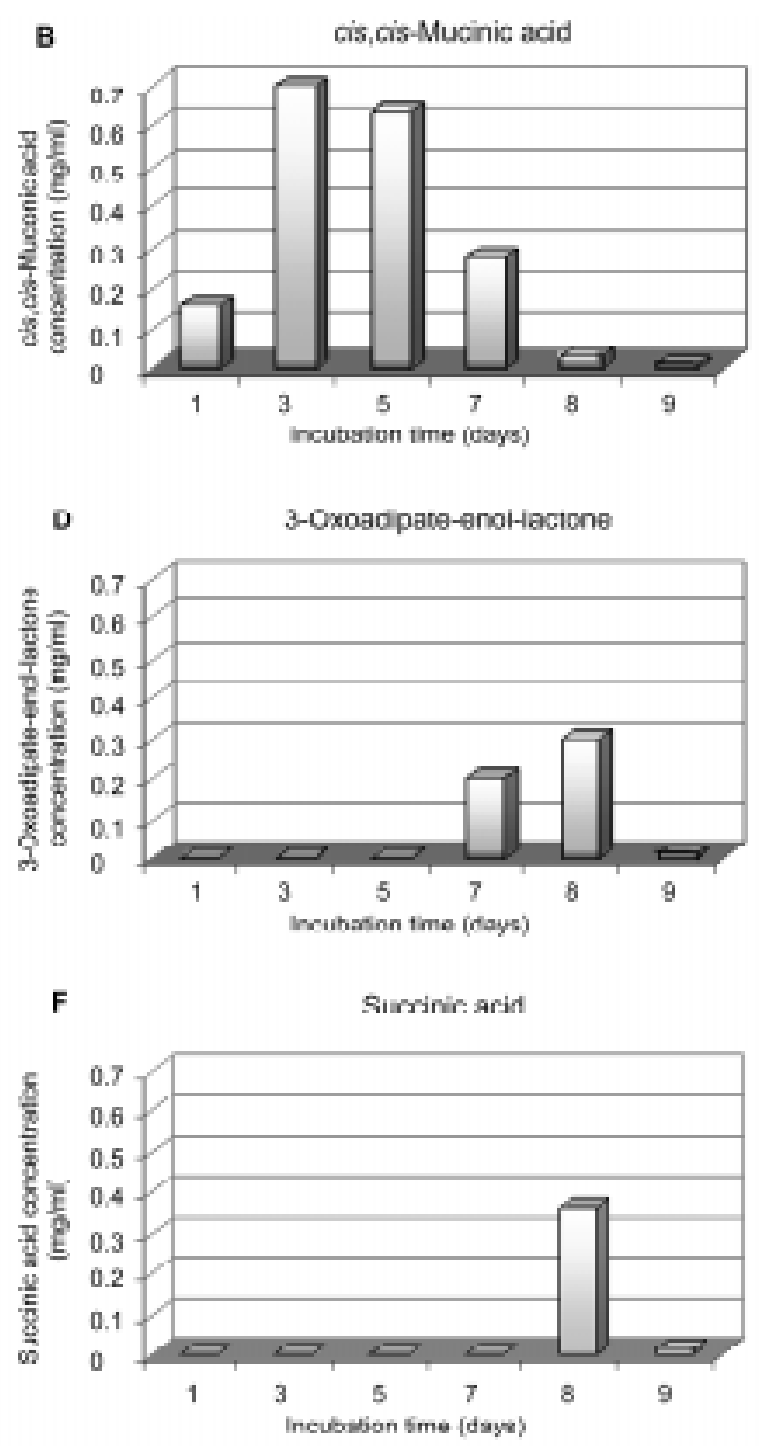

Fig. 3. HPLC determination of intermediate metabolites in cell extracts obtained on consecutive days of C. methanosorbosa BP-6 yeast culture in AY medium in the presence of aniline $(0.5 \%)$. 
catechol, cis,cis-muconic acid and cis,cis-2-hydroxymuconic-6-semialdehyde. In addition, aniline and its metabolites were determined by High Performance Liquid Chromatography (HPLC) method, with authentic samples of each metabolite serving as internal standards.

The analysis performed by photometric Spectroquant ${ }^{\circledR}$ test revealed the stepwise decrease of aniline concentration in C. methanosorbosa BP-6 yeast extract prepared from samples taken in consecutive days of fermentation (Fig. 2A). The measurements of ammonium-nitrogen (Hach UniCell ${ }^{\mathrm{TM}}$ test) showed the highest concentration of $\mathrm{NH}_{4}-\mathrm{N}$ on the day 3 and its subsequent gradual decrease (Fig. 2B). The formation of ammonium ions is clear evidence of aniline dioxygenase activity (Liu et al., 2002).

Determination of catechol was performed by spectrophotometric method according to Nair and Vaidyanathan (1964) and showed highest concentration of this metabolite in cell extract $(0.50 \mathrm{mg} / \mathrm{ml})$ on the day 5 of fermentation. An important point in discussion on the mechanism of aniline biodegradation is a way of enzymatic transformation of catechol. Its convertion by catechol 1,2-dioxygenase should lead to cis,cismuconic acid (intradiolic pathway) whereas the action of catechol 2,3-dioxygenase should give cis,cis-2hydroxymuconic-6-semialdehyde (extradiolic pathway). The levels of aforementioned metabolites in cell extracts were determined by adaptation of the procedure described by Liu et al. (2002). The measurements showed the highest concentration of both metabolites in cell extracts on day 5 of fermentation, amounting to $0.376 \mathrm{mg} / \mathrm{ml}$ for cis,cis-muconic acid and $0.053 \mathrm{mg} / \mathrm{ml}$ for cis,cis-2-hydroxymuconic-6semialdehyde. The clear domination of 1,2-dioxygenase in a cleavage of catechol strongly suggest that biodegradation of aniline by $C$. methanosorbosa BP-6 yeasts under applied conditions proceeds predominantly according to the intradiolic pathway.

In order to confirm this assumption, biodegradation of aniline by $C$. methanosorbosa BP-6 yeasts under conditions as above was followed by HPLC (GYNOTEK instrument, ODS Hypersil RP-18 column, UV-VIS detector, isocratic elution with phosphate buffer, pH $7.5-$ methanol, 60:40, v/v). Thus, the yeast cell extracts were prepared as above and, after appropriate dilution, analyzed by HPLC with authentic samples of each metabolite serving as internal standards (see Fig. 3).

The analysis revealed that all intermediates of aniline biodegradation by intradiolic pathway were present in chromatograms. The concentration of catechol was found to be growing up to the day 5 (Fig. 3A) and the level of cis,cis-muconic acid was highest between days 3 and 5 of fermentation (Fig. 3B). Muconolactone showed up between days 3 and 7 of aniline biodegradation (Fig. 3C), preceding the formation of 3-oxoadipate enol-lactone (days 7 and 8, Fig. 3D) and 3-oxoadipic acid (days 7 and 8, Fig. 3E). Finally, succinic acid was found in cell extract taken on the day 8 of fermentation (Fig. 3F). Thus, HPLC identification and the sequence of appearance of intermediate metabolites provide strong evidence that biodegradation of aniline by yeast strain $C$. methanosorbosa BP-6 in AY medium proceeds predominantly according to the intradiolic pathway.

\section{Literature}

Agency for Toxic Substances and Disease Registry. Medical Management Guidelines: aniline, ATSDR, Atlanta, GA, 2009 (http://www.atsdr.cdc.gov).

Ahtiainen J., M. Aalto and P. Pessala. 2003. Biodegradation of chemicals in a standardized test and in environmental conditions. Chemosphere 51: 529-537.

Aoki K., R. Shinke and H. Nishira. 1983. Metabolism of aniline by Rhodococcus erythropolis AN-13. Agric. Biol. Chem. 47: 1611-1616.

Aoki K., K. Ohtsuka and R. Shinke. 1984. Rapid biodegradation of aniline by Frauteria sp. ANA-18 and its metabolism. Agric. Biol. Chem. 48: 865-872.

Arjmand M. and H. Sandermann. 1985. Mineralization of chloroaniline/lignin conjugates and of free chloroanilines by the white rot fungus Phanerochaete chrysosporium. J. Agric. Food. Chem. 33: 1055-1060.

Bachofer R., F. Lingens and W. Schafer. 1975. Conversion of aniline into pyrocatechol by a Norcardia sp.: incorporation of oxygen-18. FEBS Lett. 50: 288-290.

Barnett J. A., R. W. Payne and D. Yarrow. 1984. Yeasts: characteristics and identification. Second Edition. Cambridge University Press. Cambridge, UK.

EC Joint Research Centre. Institute for Health and Consumer Protection, EU Risk Assessment Report: aniline, European Chemicals Bureau, Ispra, Italy, 2004 (http://ecp.jrc.ec.europa.eu). Emtiazi G., M. Satarii and F. Mazaherion. 2001. The ulilization of aniline, chlorinated aniline, and aniline blue as the only source of nitrogen by fungi in water. Water Res. 35: 1219-1224.

Fujii T., M. Takeo and Y. Maeda. 1997. Plasmid-encoded genes specifying aniline oxidation from Actinetobacter sp. strain YAA. Microbiology 143: 93-99.

Fukumori F. and C. P. Saint. 1997. Nucleotide sequences and regulational analysis of genes involved in conversion of aniline to catechol in Pseudomonas putida UCC22(pTDN1). J. Bacteriol. 179: 399-408.

Helm V. and H. Reber. 1979. Investigation on the regulation of aniline utilization in Pseudomonas multivorans strain An-1. Eur. J. Appl. Microbiol. Biotechnol. 7: 191-199.

Kanhg H. Y., J. J. Kukor and K. H. Oh. 2000. Characterization of strain HY99, a novel microorganism capable of aerobic and anaerobic degradation of aniline. FEMS Microbiol. Lett. 190: 215-222. King E. O., M. K. Ward and D. E. Raney. 1954. Two simple media for the demonstration of pyocyanin and fluorescein. J. Lab. Clin. Med. 44: 301-307.

Kremer S. and O. Sterner. 1996. Metabolism of 3,4-dichloroaniline by the basidiomycete Filoboletus species TA9054. J. Agric. Food. Chem. 44: 1155-1159.

Liu Z., H. Yang, Z. Huang, P. Zhou and S. J. Liu. 2002. Degradation of aniline by newly isolated, extremely aniline-tolerant Delftia sp. AN3. Appl. Microbiol. Biotechnol. 58: 679-682. 
Lyons C. D., S. Katz and R. Bartha. 1984. Mechanisms and pathways of aniline elimination from aquatic environments. Appl. Environ. Microbiol. 48: 491-496.

Matsumura E., M. Sakai, K. Hayashi, S. Murakami, S. Takenaka and K. Aoki. 2006. Constitutive expression of catABC genes in the aniline-assimilating bacterium Rhodococcus species AN-22: production, purification, characterization and gene analysis of CatA, CatB and CatC. Biochem. J. 393: 219-226.

McClure N.C. and W.A. Venables. 1986. Adaptation of Pseudomonas putida $\mathrm{mt}-2$ to growth on aromatic amines. J. Gen. Microbiol. 132: 2209-2218.

Nair P. and C.S. Vaidyanathan. 1964. A colorimetric method for determination of pyrocatechol and related substances. Anal. Biochem. 7: 315-321.

Surovtseva E.G. and A.I. Volnova. 1972. Aniline as the sole source of carbon, nitrogen and energy for Alcaligenes faecalis. Microbiology 49: 49-53.

Suye S., A. Ogawa, S. Yokoyama and A. Obayashi. 1990. Screening and identification of Candida methanosorbosa as alcohol oxidase-producing methanol using yeast. Agric. Biol. Chem. 54: $1297-1298$
Takeo M., T. Fujii, K. Takenaka and Y. Maeda. 1998. Cloning and sequencing of a gene for the meta-cleavage pathway of aniline degradation in Acinetobacter sp. strain YAA. 1998. J. Ferment. Bioeng. 85: 514-517.

Thomas S.M. and S.W. Peretti. 1998. Continous culture dynamics for aniline metabolism by Pseudomonas sp. CIT1. Biotechnol. Bioeng. 58: 1-12.

Toräng L., P. Reuschenbach, B. Müller and N. Nyholm. 2002. Laboratory shake flask batch test can predict field biodegradation of aniline in the Rhine. Chemosphere 49: 1257-1265.

US Environmental Protection Agency. OPPT Chemical Fact Sheets, Aniline Fact Sheet, Washington, DC, 1994 (http://www. epa.gov/chemfact).

Wang L., S. Barrington and J. W. Kim. 2007. Biodegradation of pentyl amine and aniline from petrochemical wastewater. J. Environ. Manage. 83: 191-197.

Zeyer J., A. Wasserfallen and K.N. Timmis. 1985. Microbial mineralization of ring-substituted anilines through an ortho-cleavage pathway. Appl. Environ. Microbiol. 50: 447-453.

Zissi U., G. Lyberatos and S. Pavlou. 1997. Biodegradation of $p$-aminobenzene by Bacillus sublilis under aerobic conditions. J. Ind. Microbiol. Biotechnol. 19: 49-55. 